\title{
Carcinoma Microsatellite Instability Status as a Predictor of Benefit from Fluorouracil-Based Adjuvant Chemotherapy for Stage II Rectal Cancer
}

\author{
Liu Yang ${ }^{1 \star}$, Yan Sun ${ }^{2 \&}$, Xin-En Huang ${ }^{2 *}$, Dong-Sheng Yu ${ }^{1}$, Jian-Nong Zhou ${ }^{1}$, Xin \\ Zhou ${ }^{1}$, Dong-Zheng Li ${ }^{1}$, Xin Guan ${ }^{1}$
}

\begin{abstract}
Purpose: Rectal cancers with high microsatellite-instable have clinical and pathological features that differentiate them from microsatellite-stable or low- frequency carcinomas, which was studied rarely in stage II rectal cancer, promoting the present investigation of the usefulness of microsatellite-instability status as a predictor of the benefit of adjuvant chemotherapy with fluorouracil in stage II rectal cancer. Patients and Methods: Data of 460 patients who underwent primary anterior resection with a double stapling technique for rectal carcinoma at a single institution from 2008 to 2012 were retrospectively collected. All patients experienced a total mesorectal excision (TME) operation. Survival analysis were analyzed using the Cox regression method. Results: Five-year rate of disease-free survival (DFS) was noted in $390(84.8 \%)$ of 460 patients with stage II rectal cancer. Of 460 tissue specimens, $97(21.1 \%)$ exhibited high-frequency microsatellite instability. Median age of the patients was $65(50-71)$ and $185(40.2 \%)$ were male. After univariate and multivariate analysis, microsatellite instability $(p=0.001)$, female sex $(p<0.05)$ and fluorouracil-based adjuvant chemotherapy $(p<0.001)$, the 3 factors were attributed to a favorable survival status independently. Among 201 patients who did not receive adjuvant chemotherapy, those cancers displaying high-frequency microsatellite instability had a better 5-year rate of DFS than tumors exhibiting microsatellite stability or low-frequency instability (HR, 13.61 [95\% CI, 1.88 to 99.28]; $p=0.010$ ), while in 259 patients who received adjuvant chemotherapy, there was no DFS difference between the two groups $(p=0.145)$. Furthermore, patients exhibiting microsatellite stability or low-frequency instability who received adjuvant chemotherapy had a better 5-year rate of DFS than patients did not (HR, 5.16 [95\% CI, 2.90 to 9.18]; $p<0.001$ ), while patients exhibiting high-frequency microsatellite instability were not connected with increased DFS $(p=0.696)$. It was implied that female patients had better survival than male. Conclusion: Survival status after anterior resection of rectal carcinoma is related to the microsatellite instability status, adjuvant chemotherapy and gender. Fluorouracil-based adjuvant chemotherapy benefits patients of stage II rectal cancer with microsatellite-stable or low microsatellite-instable, but not those with high microsatelliteinstable. Additionally, free of adjuvant chemotherapy, carcinomas with high microsatellite-instable have a better 5-year rate of DFS than those with microsatellite-stable or low microsatellite-instable, and female patients have a better survival as well.
\end{abstract}

Keywords: Rectal cancer - microsatellite instability - survival analysis - adjuvant chemotherapy

Asian Pac J Cancer Prev, 16 (4), 1545-1551

\section{Introduction}

It has been found that $\mathrm{CRC}$ is the third commonest cancer in males and the second in females. In 2008, more over 1.2 million new CRC cases and 608,700 deaths were reckoned to be occurred (Ahmedin et al., 2011; Chen et al., 2013; Chen et al., 2014). With the advent of stapling devices, surgical operation combining with preoperative chemo-radiation therapy (PCRT) and by means of total mesorectal excision (TME), which makes for a high rate of anal sphincter preservation in present days (Tjandra et al., 2005). As surgical procedures independent is possibly sanative, plenty of sufferers suffer recurrences of local or remote, and those with the highest recurrence rate are suggested to receive fluorouracil-based systemic adjuvant chemotherapy, which has been proven to be beneficial in a lot of previous trials and analyses (Thierry et al., 2009; Yang et al., 2013 ; Yang et al., 2013 ; Gong et al., 2014). In sporadic CRC, carcinogenesis can be subdivided into two main groups defined by two different mechanisms. The first, termed chromosomal instability accounts for around $80 \%$ of CRC, is associated with loss of heterozygosity 
Table 1. Demographic Data and Clinical Characteristics of the Selected Samples Stratified by Groups

\begin{tabular}{|c|c|c|c|c|}
\hline Variable & Categories & Survival $(n=390)$ & Recurrence or death $(n=70)$ & $p$ value \\
\hline \multirow[t]{3}{*}{ Age median (IQR) y } & & $61(50-69)$ & $68(55-74)$ & \\
\hline & $<65$ & 287 & 49 & $p=0.125$ \\
\hline & $\geq 65$ & 103 & 21 & \\
\hline \multicolumn{5}{|l|}{ Gender } \\
\hline & Male & 161 & 24 & $p<0.05$ \\
\hline & Female & 299 & 46 & \\
\hline \multicolumn{5}{|c|}{ Physical status (ASA score) } \\
\hline & $\mathrm{ASA} \leq 2$ & 335 & 62 & $p=0.219$ \\
\hline & $\mathrm{ASA} \geq 3$ & 55 & 8 & \\
\hline \multicolumn{5}{|c|}{ Microsatellite instability status } \\
\hline & Microsatellite stability & 297 & 66 & $p=0.005$ \\
\hline & Microsatellite instability & 93 & 4 & \\
\hline \multicolumn{5}{|l|}{ Tobacco abuse } \\
\hline & $\leq 4$ cigarettes per day & 206 & 38 & $p=0.505$ \\
\hline & $\geq 5$ cigarettes per day & 184 & 32 & \\
\hline \multicolumn{5}{|l|}{ Alcohol abuse } \\
\hline & $\leq 2$ glasses per day & 257 & 44 & $p=0.751$ \\
\hline & $\geq 3$ glasses per day & 133 & 26 & \\
\hline \multicolumn{5}{|c|}{ Tumor site (from the anal verge) } \\
\hline & $<4 \mathrm{~cm}$ & 75 & 11 & $p=0.923$ \\
\hline & $\geq 4 \mathrm{~cm}$ & 315 & 59 & \\
\hline \multicolumn{5}{|l|}{ Preoperative BMI } \\
\hline & $<35$ & 360 & 65 & $p=0.08$ \\
\hline & $\geq 35$ & 30 & 5 & \\
\hline \multicolumn{5}{|c|}{ Fluorouracil-based adjuvant chemotherapy } \\
\hline & yes & 241 & 18 & $p<0.001$ \\
\hline & no & 149 & 52 & \\
\hline \multicolumn{5}{|l|}{ Diabetes mellitus } \\
\hline & without & 369 & 66 & $p=0.522$ \\
\hline & with & 21 & 4 & \\
\hline
\end{tabular}

Abbreviations: IQR, interquartile range

of tumor suppressor genes and frequent mutations in APC, KRAS and TP53. On the other hand, a subset makes up roughly $15 \%$ of CRC proves DNA mismatch repair (MMR) deficiency, characterized by extensive microsatellite instability as a result from defective DNA mismatch repair (MMR) system (Lengauer et al., 1997; Kuismanen et al., 2000; Grady et al., 2008).

Most of microsatellite instability cancers are sporadic cause of the promoter region of the mismatch repair gene, MLH1, is silenced by $\mathrm{CpG}$ island hypermethylation (Cunningham et al., 1998). In condition, microsatellite instability is also a distinct feature of Lynch syndrome, as the consequence of a germ line mutation in a MMR gene (Lynch et al., 2009). Detection of microsatellite instability demands DNA extraction from cancer and normal tissue, which can be technically challenging and time consuming. Multiple methods, such as immunohistochemistry analysis, histopathologic evaluation, microsatellite analysis and DNA ploidy analysis are proposed to be used as an integration (Lanza et al., 2006).

Undoubtedly, colorectal cancers displaying high microsatellite-instable have been related to a larger size of the primary tumor, a more favorable stage distribution, and a longer survival than those stage-matched microsatellite stability cancers (Kim et al., 1994). As efforts have been made to improve outcome in stage II colorectal cancer patients where 5-year survival is already approximately 80\% (Midgley et al., 2005), few investigations have inspected the outcomes of adjuvant treatment in stage
II rectal cancers with different microsatellite instability status. Moreover, many surveys that have conducted for the effects of adjuvant therapy, which have already gotten populations with latent biases (Halling et al., 1999; Gryfe et al., 2000; Hemminki et al., 2000), and lack of data about the outcomes of stage II rectal cancers from a single-institute of one team of surgeons.

Specimens from stage II rectal cancer patients underwent anterior resection were used in the this study, which were previously enrolled in retrospective, randomized trials of fluorouracil-based chemotherapy. Additionally, the main objective of studying this population of patients was to analyze if microsatellite instability status of tumor could be an independent predictor of a benefit from fluorouracil-based adjuvant chemotherapy in stage II rectal cancer.

\section{Materials and Methods}

Between January 2008 and December 2012, 460 randomly collected and routinely followed up patients with rectal cancer underwent anterior resection with double stapling anastomosis for primary rectal cancer at Colorectal Cancer Center, the Affiliated Jiangsu Cancer Hospital of Nanjing Medical University \& Jiangsu Institute of Cancer Research, Nanjing, China. The medical notes of those patients were reviewed in detail. Eligibility criteria included rectal cancer, phase II of TNM stage, with adjuvant radiotherapy, histologically 
Table 2. Multivariate Analysis about DFS of All Patients by Cox Regression

\begin{tabular}{|c|c|c|c|c|}
\hline \multirow{2}{*}{$\begin{array}{l}\text { Factors } \\
\text { Lower } \\
\text { High-microsatellite } \\
\text { instable }\end{array}$} & \multirow{2}{*}{$\begin{array}{r}p \text { value } \\
\text { Upper } \\
0.005\end{array}$} & \multirow{2}{*}{$\begin{array}{c}\text { HR } \\
4.334\end{array}$} & \multicolumn{2}{|c|}{$95 \% \mathrm{CI}$ for $\mathrm{HR}$} \\
\hline & & & 1.557 & 12.064 \\
\hline $\begin{array}{l}\text { With fluorouracil-based } \\
\text { adjuvant chemotherapy }\end{array}$ & 0 & 4.252 & 2.484 & 7.278 \\
\hline Male sex & 0.049 & 0.607 & 0.368 & 1 \\
\hline
\end{tabular}

proven adenocarcinoma, open and laparoscopic surgery with pelvic drainage, antibiotics using for 7 postoperative days, and cancer-related decease. Exclusion criteria were as follows: Hartmann's and Miles' procedure, without adjuvant radiotherapy, phase I, III and IV of TNM stage, colon cancer, hand-sewn anastomosis, lost observation and disease-free death. Rectal cancer was classified according to the distance from the anal verge, as determined by rigid
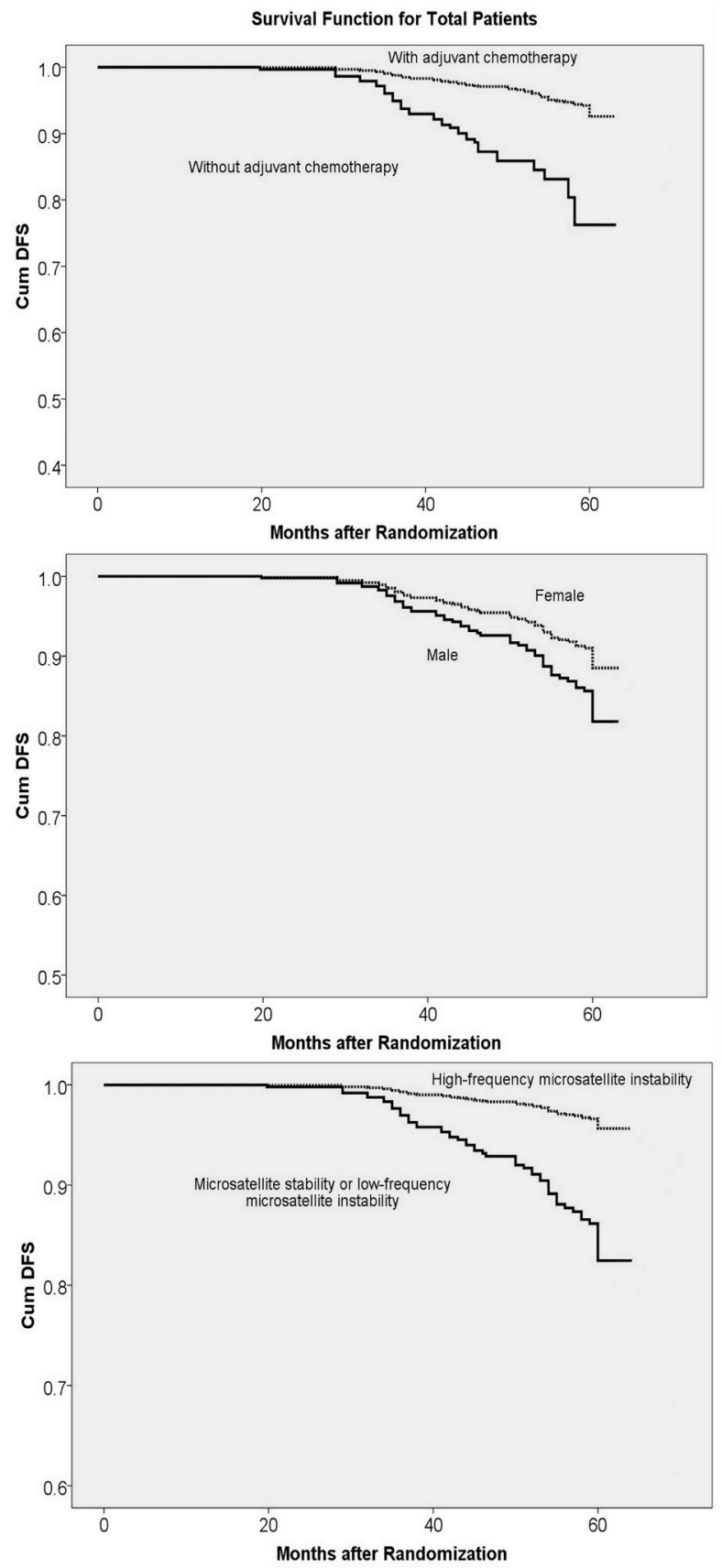

Figure 1 sigmoidoscopy. TME was adopted as the standard surgical technique according to tumor location. Blocks of formalinfixed, paraffin-embedded specimens were requested from the relevant pathology departments. Various independent clinical variables were analyzed and detailed in Table 1. The Ethics Committee of Science approved data collection in the register.

Tissue samples from tumor and normal rectal mucosa were obtained from each patient and DNA was extracted from them. In those cases in which fresh tissue was not available, archival formalin fixed, paraffin embedded samples were used. Genomic DNA was isolated using the Qia Amp Tissue Kit (Qiagen, Duesseldorf, Germany). Microsatellite instability status was assessed using the BAT-25, BAT-26, D5S346, D2S123 and D17S250, the 5 mononucleotide markers (National Cancer Institute, 1997), based on their high sensitivity as markers for cancer microsatellite instability status, thus differences less than 2 markers between cancer and normal tissue groups were defined as microsatellite-stable or low microsatelliteinstable, and vice versa. Primers were fluorescently labelled and analysed on an ABI 310 Genetic Analyser using GeneScan Analysis software (Applied Biosystems, Foster City, California, USA).

The patient was placed in a modified lithotomy, right side down, Trendelenburg position. For patients underwent laparoscopic surgery, an initial 12-mm port placement was carried out using the open technique, and pneumoperitoneum was accomplished using carbon

Survival Function for Patients with Adjuvant Chemotherapy

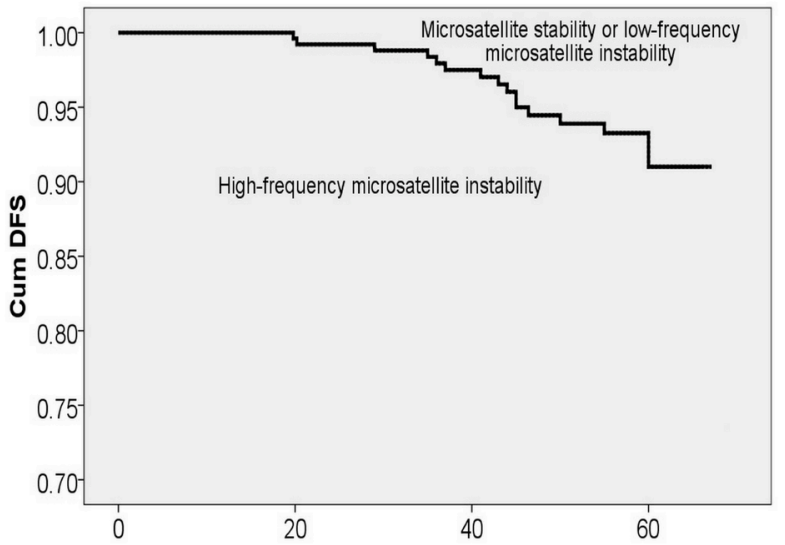

Survival Function for Patients without Adjuvant Chemotherapy

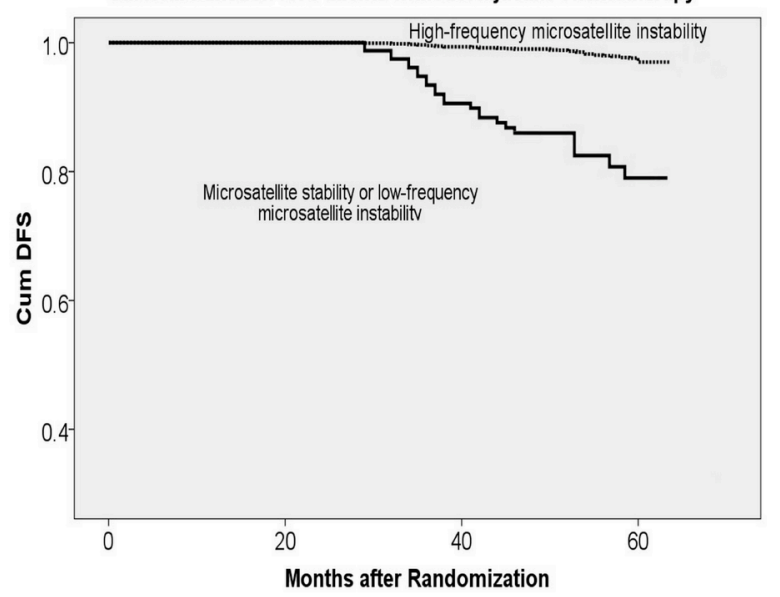

Figure 2 

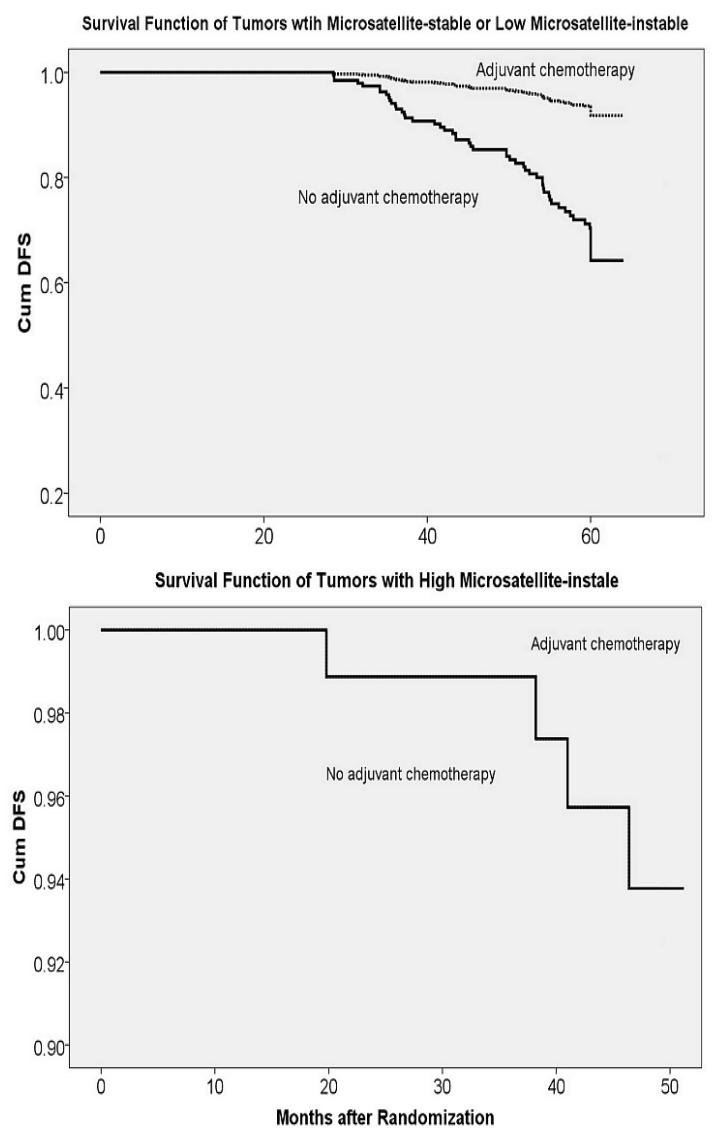

Figure 3

dioxide. A standard 10-mm laparoscope was inserted through the 12-mm trocar, and then two 5-mm ports were inserted in the upper right and left abdominal quadrants and two more 12-mm ports were placed in the lower right and left abdominal quadrants under laparoscopic guidance. For patients underwent open surgery, a median incision in lower abdomen was made to expose surgical field.

Patients were followed up routinely by a protocol consisting of visits every 3 months for the first 2 years, followed by visits every 6 months for the next 3 years. CEA, CA242, CA724 and CA199 levels were reviewed at each visit, and a CT scan of abdomen, pelvis, and thorax was performed at the every half-year follow-up. Colonoscopy was performed 1 year after surgery when the colon and rectum had previously been cleared of synchronous lesions, and repeated at 3-year intervals unless otherwise indicated by findings. Apart from this, patients were investigated in further detail as appropriate according clinical symptoms and findings.

Demographic, clinical, and tumor-related characteristics of patients, as well as a detail information on each donor, were obtained. Age at cancer diagnosis, gender, tumor site, tobacco and alcohol abuse, ASA status, diabetes mellitus, adjuvant chemotherapy, preoperative body mass index (BMI), procedures and microsatellite instability status were registered for each affected patient or participant. All continuous variables were dichotomized. Chi-squared or Fisher's exact test for categorical variables was used for statistical comparisons of those variables between groups. Multivariate analysis to detect risk factors for survival status was conducted with a Cox regression model. Difference in each variable has been analyzed using one- way analysis of variance (ANOVA) before multivariate analysis was performed.

Follow-up time was defined as time between initial operation and death or last observation. Although a 60-month follow-up was scheduled, median follow-up times were often less due to death. For instance, the median follow-up time for the 460 patients with rectal cancer was 41.5 months due to death and the resultant decreased follow-up period. Survival analysis were analysed using the Cox regression method. Significance was calculated at the $95 \% \mathrm{CI}$ and $p$ value $=0.05$. Statistical analyses were performed using IBM SPSS statistics 19.0 for Windows (SPSS Inc; IBM, Chicago, IL).

\section{Results}

The demographic data and clinical characteristics of the selected sample stratified by goup are detailed in Table 1. The total DFS rate was $84.8 \%$ (390/460 patients). A total of 460 patients [185 male patients (40.2\%)] with a median age of 65 (50-71) years at the time of surgery were included. The majority of patients were ASA 1 or $2(86.3 \%)$. Eighty six $(18.7 \%)$ patients presented rectal cancer within $4 \mathrm{~cm}$ from the anal verge. Among all these patients, $47.0 \%$ (216) \& $34.6 \%$ (159) of whom abused tobacco and alcohol, respectively. Twenty five (5.4\%) sufferers were diagnosed with diabetes mellitus preoperatively. The preoperative BMI was equal or greater than 35 (severe obesity) in $35(7.6 \%$ ) patients. The relationship of demographic data, clinical charateristics and survival status are summarized in Table 1 and Figure 1.

After univariate and multivariate analysis, microsatellite instability $(p=0.001)$, microsatellite instability $(p=0.001)$, fluorouracil-based adjuvant chemotherapy $(p<0.001)$ and male sex $(p<0.05)$, as the former two brought about a favorable DFS independently, the latter one had a negative impact on DFS (Table 2 and Figure 1). Assessments testified that no interaction was found in effect of chemotherapy and microsatellite instability status, or both differed in terms of genders, and hence, patients were collected regardless of gender for the analyses examining chemotherapy effect and microsatellite-instability status. Among 201 patients who did not receive adjuvant chemotherapy, those cancers displaying high-frequency microsatellite instability had a better 5-year rate of DFS than tumors exhibiting microsatellite stability or lowfrequency instability (HR, 13.61 [95\% CI, 1.88 to 99.28]; $p=0.010$ ) (Figure 2), while in 259 patients who received adjuvant chemotherapy, there was no DFS difference between the two groups $(p=0.145)$. Furthermore, patients exhibiting microsatellite stability or low-frequency instability, which received adjuvant chemotherapy had a better 5-year rate of DFS than patients did not (HR, 5.16 [95\% CI, 2.90 to 9.18]; $p<0.001$ ), while patients exhibiting high-frequency microsatellite instability were not connected with increased DFS ( $p=0.696)$ (Figure 3).

\section{Discussion}

Although adequate clinical data that boost the 
utilization of adjuvant chemotherapy in stage II colorectal cancer and varieties of researches disclose a corresponding proportional decline in the probability of recurrence, there is no absolutely clear proof can affirm that all stage II patients should exactly receive adjuvant chemotherapy, as well as lack of investigation as for stage II rectal cancer from a single-team, thus the merit and demerit should be preferred to patients to let them make an informed choice, And it is reported that, however, only around 5\% benefit observed applies to the stage II population by and large, some previous studies exposes better predictive markers for chemotherapeutic response (Midgley et al., 2005).

In this manuscript, all objects incorporated a retrospective collection of tissue and germline DNA for assessing both prognostic markers and that for chemotherapeutic response were performed consecutively and randomly, on the way of individualizing therapy.

As for DNA testing, it was newly confirmed that colorectal cancer has two molecular pathways resulting in the malignant phenotype, the pathway of high microsatellite-instable and that of microsatellite-stable, which respond dissimilarly to DNA damage. Consequently, it is impossible that tumors with these different pathways would react equally to chemotherapeutic agents that damage DNA (Ribic et al., 2003).

In this series, to restrain patients of potentially curable advanced-stage colorectal cancer from chemotherapy in a clinical trial may not be ethical, therefore cases from a single-center, randomized and controlled trial was selected to ascertain if microsatellite-instable status could act as a predictor of a DFS benefit from fluorouracil-based adjuvant chemotherapy. And the advantages of a single register include the sufficient number of suffers which can be collected data based on a truly unselected study population and without biases or confounding factor (Yang et al., 2013).

Meta analysis showed that colorectal cancer with high microsatellite instable have a significantly better prognosis compared to those with intact mismatch repair (Popat et al., 2005), which is consist with our outcomes that the patients with tumor exhibiting high microsatellite-instable had a better DFS than those with tumor exhibiting low microsatellite-instable or microsatellite-stable $(p=0.001)$.

Besides that, it was found that among 201 patients who did not receive adjuvant chemotherapy, those cancers with high microsatellite-instable have a better 5-year rate of DFS than tumors with microsatellite-stable or low microsatellite-instable $(p=0.010)$.

Theoretically, the reason for above could be argued that the term of the $\mathrm{CpG}$ Island Methylator Phenotype (CIMP) is necessary to a crucial subset of colorectal cancer, which links with microsatellite instability and also detects in BRAF mutant microsatellite stable cancers that are involved in poor prognosis. To clarify further, a recent study revealed that the isocitrate dehydrogenase 1 (IDH1) mutation as if gives rise to CIMP in a small proportion of BRAF mutant owing to an activating mutation that generates the 2-hydroxyglutarate oncometabolite in microsatellite stable colorectal cancers, thus it offers a brand new foretype that a single gene mutation may cause CIMP in colorectal cancer, and that will lead to a particular epigenetic signature and clinicopathological features (Whitehall et al., 2014).

Unexpectedly, there were something contrary to our results that the relationship of hypomethylation in long intersperses nucleotide element-1 (LINE-1) and high microsatellite-instable in colorectal cancer to worse and better survival has been noticed already (Ahn et al., 2011). The relationship of LINE-1 hypomethylation to worse survival is stronger in high microsatellite-instable colorectal cancers than in microsatellite-stable colorectal cancers, thus cancer LINE-1 methylation level may be a helpful survival biomarker to identify invasive carcinomas among high microsatellite-instable colorectal cancers (Inamura et al., 2014), and that needs our further study.

In the present study, there was no DFS difference between the two different microsatellite status groups of those 259 patients who received adjuvant chemotherapy $(p=0.145)$. Therefore, compared with the 201 patients without adjuvant chemotherapy above, the outcomes of the patients with chemotherapy may reflect the fact that a fluorouracil-based adjuvant chemotherapy could benefit the stage II rectal cancer patients with low microsatelliteinstable or microsatellite-stable, but could not profit the patients with high microsatellite-instable, and that was demonstrated particularly thereinafter. Reportedly, the high microsatellite-instable is not only a good prognostic factor, but also a predictive factor of non-chemotherapyresponse (Des Guetz et al., 2009), which is in keeping with our findings.

It was also found that patients exhibiting microsatellite stability or low-frequency instability who received adjuvant chemotherapy likely benefit from fluorouracilbased adjuvant chemotherapy, $(p<0.001)$, whereas such chemotherapy did not benefit patients with high microsatellite-instable and may in fact have led to worse outcomes among such patients, which was portrayed in Figure 3 ( $p=0.696)$.

As proved in previous studies, it is clear that patients with stage II colorectal cancer have a DFS benefit from fluorouracil-based adjuvant chemotherapy, but only for tumors with microsatellite-stable (Thibodeau et al., 1993; Halling et al., 1999; Gryfe et al., 2000; Wright et al., 2000; Midgley et al., 2005). Is there any agent or regimen to improve the DFS of stage II rectal cancers exhibiting high microsatellite-instable? A prospective, multicentric, controlled and randomized clinical trial answered the question, which implies that sufferers with high microsatellite-instable treated with the combination of irinotecan and fluorouracil may have a better survival than those who received fluorouracil-based adjuvant chemotherapy only (Bertagnolli et al., 2009).

It is surprising that the findings displayed a DFS difference between sexes in our research, which indicated that female patients had a better DFS than males despite the tumor microsatellite instability status and whether or not receiving adjuvant chemotherapy $(p<0.05)$.

On the basis of a recent literature, it was demonstrated that sex steroids, the oestrogen receptor $b(\mathrm{ERb})$, is the predominantly expressed ER in the colorectum and loss of ERb in CRC had been associated with advanced cancer stages; additionally, lack of ERb expression led 
to advanced cancer stages and independently associated with poor survival (Rudolph et al., 2012). Another study suggested that low 17beta-hydroxysteroid dehydrogenase (17HSD) type 2 mRNA expression is an independent marker of favorable prognosis in females with distal colorectal cancer, supporting the presence of genderand location-related differences in the pathogenesis of colorectal cancer (Oduwole et al., 2003). As mentioned above, female sex may harbor an increased DFS of stage II rectal cancer patients, which is in line with our outcomes.

Current study is a retrospective analysis of a large singcenter database with limitations. Coding for comorbidities and complications may make for biased results due to the vague nature of ICD-9 definitions for the variables. As this was not a randomised study, other factors may have further contributed to the better efficacy of fluorouracilbased regimen, have not been investigated in our study. Similarly, as surgery has a major impact on the treatment of rectal cancer at early stages, improved surgical techniques might have contributed to the longer DFS of patients given agents, which may makes for biases. This retrospective review, however, is one of the largest and most comprehensive studies investigating the DFS of stage II rectal cancer after anterior resection, which is affected by cancer microsatellite instability status.

To sum up, a favorable survival status after anterior resection of rectal carcinoma may be related to the high microsatellite-instable, receiving fluorouracil-based adjuvant chemotherapy and female sex, independently and respectively. Fluorouracil-based adjuvant chemotherapy benefits patients of stage II rectal cancer with microsatellitestable or low microsatellite-instable, but not those with high microsatellite-instable. In addition, free of adjuvant chemotherapy, carcinomas with high microsatelliteinstable have a better 5-year rate of DFS than those with microsatellite-stable or low microsatellite-instable, and female patients may have a favorable prognosis as well.

\section{Acknowledgements}

The authors declare that there is no conflict of interest with any one that could bias the work.

\section{References}

Ahmedin J, Freddie B, Melissa MC, et al (2011). Global cancer statistics. Ca Cancer J Clin, 61, 69-90.

Ahn JB, Chung WB, Maeda O, et al (2011). DNA methylation predicts recurrence from resected stage III proximal colon cancer. Cancer, 117, 1847-54.

André T, Boni C, Navarro M, et al (2009). Improved overall survival with oxaliplatin, fluorouracil, and leucovorin as adjuvant treatment in stage II or III colon cancer in the MOSAIC trial. J Clin Oncol, 27, 3109-16.

Bertagnolli MM, Niedzwiecki D, Compton CC, et al (2009). Microsatellite instability predicts improved response to adjuvant therapy with irinotecan, fluorouracil, and leucovorin in stage III colon cancer: Cancer and Leukemia Group B Protocol 89803. J Clin Oncol, 27, 1814-21.

Chen YS, Xu SX, Ding YB, et al (2013). Helicobacter pylori Infection and the risk of colorectal adenoma and adenocarcinoma: an updated meta-analysis of different testing methods. Asian Pac J Cancer Prev, 14, 7613-9.

Chen YS, Xu SX, Ding YB, et al (2014). Colorectal cancer screening in high-risk populations: a survey of cognition among medical professionals in Jiangsu, China. Asian Pac J Cancer Prev, 14, 6487-91.

Cunningham MJ, Christensen ER, Tester DJ, et al (1998). Hypermethylation of the hMLH1 promoter in colon cancer with microsatellite instability. Cancer Res, 58, 3455-60.

Des Guetz G, Schischmanoff O, Nicolas P, et al (2009). Does microsatellite instability predict the efficacy of adjuvant chemotherapy in colorectal cancer? A systematic review with meta-analysis. Eur J Cancer, 45, 1890-6.

Gong JP, Yang L, Huang XE, et al (2014). Outcomes based on risk assessment of anastomotic leakage after rectal cancer surgery. Asian Pac J Caner Prev, 15, 707-12.

Grady WM, Carethers JM. Genomic and epigenetic instability in colorectal cancer pathogenesis (2008). Gastroenterology, 135, 1079-99.

Gryfe R, Kim H, Hsieh ETK, et al (2000). Tumor microsatellite instability and clinical outcome in young patients with colorectal cancer. N Engl J Med, 342, 69-77.

Halling KC, French AJ, McDonnell SK, et al (1999). Microsatellite instability and 8p allelic imbalance in stage B2 and C colorectal cancers. J Natl Cancer Inst, 91, 1295-303.

Hemminki A, Mecklin JP, Jarvinen H, et al (2000). Microsatellite instability is a favorable prognostic indicator in patients with colorectal cancer receiving chemotherapy. Gastroenterology, 119, 921-8

Inamura K, Yamauchi M, Nishihara R, et al (2014). Tumor LINE-1 methylation level and microsatellite instability in relation to colorectal cancer prognosis. J Natl Cancer Inst, 106, dju195.

Kim H, Jen J, Vogelstein B, et al (1994). Clinical and pathological characteristics of sporadic colorectal carcinomas with DNA replication errors in microsatellite sequences. Am J Pathol, 145, 148-56.

Kuismanen SA, Holmberg MT, Salovaara R, et al (2000). Genetic and epigenetic modification of MLH1 accounts for a major share of microsatellite-unstable colorectal cancers. Am J Pathol, 156, 1773-9.

Lanza G, Gafà R, Santini A, et al (2006). Immunohistochemical test for MLH1 and MSH2 expression predicts clinical outcome in stage II and III colorectal cancer patients. J Clin Oncol, 24, 2359-67.

Lengauer C, Kinzler KW, Vogelstein B (1997). Genetic instability in colorectal cancers. Nature, 386, 623-7.

Lynch HT, Lynch PM, Lanspa SJ, et al (2009). Review of the Lynch syndrome: history, molecular genetics, screening, differential diagnosis, and medicolegal ramifications. Clin Genet, 76, 1-18.

Midgley R, Kerr DJ (2005). Adjuvant chemotherapy for stage II colorectal cancer: the time is right! Nat Clin Pract Oncol, 2, 364-9.

Popat S, Hubner R, Houlston RS (2005). Systematic review of microsatellite instability and colorectal cancer prognosis. $J$ Clin Oncol, 23, 609-18.

Ribic CM, Sargent DJ, Moore MJ, et al. Tumor microsatelliteinstability status as a predictor of benefit from fluorouracilbased adjuvant chemotherapy for colon cancer (2003). N Engl J Med, 349, 247-57.

Rudolph A, Toth C, Hoffmeister M, et al (2012). Expression of oestrogen receptor $\beta$ and prognosis of colorectal cancer. $\mathrm{Br}$ J Cancer, 107, 831-9.

Thibodeau SN, Bren G, Schaid D (1993). Microsatellite instability in cancer of the proximal colon. Science, $\mathbf{2 6 0}$, 816-9.

Oduwole OO, Mäkinen MJ, Isomaa VV, et al (2003). 17Beta- 
hydroxysteroid dehydrogenase type 2: independent prognostic significance and evidence of estrogen protection in female patients with colon cancer. J Steroid Biochem Mol Biol, 87, 133-40.

Tjandra JJ, Kilkenny JW, Buie WD, et al (2005). Standards practice task force; American society of colon and rectal surgeons. Practice parameters for the management of rectal cancer (revised). Dis Colon Rectum, 48, 411-23.

Whitehall V, Dumenil T, McKeone D, et al (2014). Isocitrate dehydrogenase $1 \mathrm{R} 132 \mathrm{C}$ mutation occurs exclusively in microsatellite stable colorectal cancers with the $\mathrm{CpG}$ island methylator phenotype. Epigenetics, 9, 1454-60.

Wright CM, Dent OF, Barker M, et al (2000). Prognostic significance of extensive microsatellite instability in sporadic clinicopathological stage C colorectal cancer. Br J Surg, 87, 1197-202.

Yang L, Huang XE, Zhou JN (2013). Risk assessment on AL after rectal cancer surgery: an analysis of 753 patients. Asian Pac J Cancer Prev, 14, 4447-53.

Yang L, Huang XE, Xu L, et al (2013). Acidic pelvic drainage as a predictive factor for AL after surgery for patients with rectal cancer. Asian Pac J Cancer Prev, 14, 5441-7. 